\title{
An analysis of temperatures and wind speeds above Dome C, Antarctica
}

\author{
E. Aristidi ${ }^{1}$, K. Agabi ${ }^{1}$, M. Azouit ${ }^{1}$, E. Fossat ${ }^{1}$, J. Vernin ${ }^{1}$, T. Travouillon ${ }^{2}$, J. S. Lawrence ${ }^{2}$, C. Meyer ${ }^{2}$, \\ J. W. V. Storey ${ }^{2}$, B. Halter ${ }^{3}$, W. L. Roth ${ }^{3}$, and V. Walden ${ }^{3}$ \\ ${ }^{1}$ Laboratoire Universitaire d'Astrophysique de Nice, Université de Nice Sophia Antipolis, Parc Valrose, \\ 06108 Nice Cedex 2, France \\ e-mail: aristidi@unice.fr \\ 2 School of Physics, University of New South Wales, Sydney, NSW 2052, Australia \\ e-mail: tonyt@phys.unsw.edu.au \\ 3 Department of Geography, University of Idaho, Moscow, Idaho, USA
}

Received 21 August 2004 / Accepted 24 September 2004

\begin{abstract}
A good astronomical site must fulfill several criteria including low atmospheric turbulence and low wind speeds. It is therefore important to have a detailed knowledge of the temperature and wind conditions of a location considered for future astronomical research. Antarctica has unique atmospheric conditions that have already been exploited at the South Pole station. Dome C, a site located on a local maximum of the Antarctic plateau, is likely to have even better conditions. In this paper we present the analysis of two decades of wind speed measurements taken at Dome $\mathrm{C}$ by an automated weather station (AWS). We also present temperature and wind speed profiles taken over four Antarctic summers using balloon-borne weather sondes. We will show that as well as having one of the lowest average wind speed ever recorded at an existing or potential observatory, Dome $\mathrm{C}$ also has an extremely stable upper atmosphere and a very low inversion layer.
\end{abstract}

Key words. site testing - atmospheric effects - balloons

\section{Introduction}

Located at an altitude of $3250 \mathrm{~m}$, on a local maximum of the Antarctic plateau, Dome C is currently the subject of an intense site testing campaign led by the University of Nice and the University of New South Wales. Already showing excellent sky transparency in the sub-millimeter (Calisse et al. 2004) and very good day time seeing (Aristidi et al. 2003), the site is predicted to offer better transparency and image resolution than any other known site in the world across the whole range of usable wavelengths.

An important description of the site is contained in the wind and temperature profiles through the atmosphere. These two factors influence several aspects of telescope design and performance at a given site. Temperature and wind speed gradients result in the formation of optical turbulence. The spatial and temporal stability of these quantities in turn determine the performance of Adaptive Optics (AO) systems. The wind speed determines the rate at which the turbulence is blown across the aperture of the telescope. Under the "frozen screen" (Taylor) hypothesis, the time constants that are relevant to adaptive optics correction are simply related to the time required to move a turbulent atmospheric cell across its own diameter. Low wind speeds imply long time constants. This not only reduces the bandwidth necessary for control of the deformable mirror, but also increases the integration time that is available on the guide star. In turn, this leads either to an increased accuracy of correction, the ability to use fainter guide stars, or both.

The thickness and intensity of the turbulent ground layer usually increases with wind speed. At the Uzbek observatory site of Mt Maidanak, where the seeing contribution of the boundary layer is important there is a clear positive correlation (Ehgamberdiev et al. 2000), while at South Pole the relationship is so strong that it is almost a linear dependence (Travouillon et al. 2003a). This is to be expected as the Antarctic plateau is essentially free of any topographic features. The energy content of the turbulent ground layer is therefore expected to be directly related to the energy of the local wind that is generating it.

Finally, greater wind speeds result in greater levels of dust (or, in the case of Antarctica, ice crystals). Lower wind speeds imply cleaner optical surfaces and a better overall operating environment for the mechanical systems, electronics, and observer.

In this paper we present the results of wind and temperature profiles measured by balloon-borne weather sondes during four Antarctic summers between 2000 and 2004 as well as the ground wind measurements taken over the past 20 years by an 
automated weather station (AWS). A complete statistical analysis is presented along with a comparison to other astronomical sites. Finally, a Richardson turbulence generation analysis is presented in order to derive the potential turbulence conditions of the site.

\section{Data acquisition}

\subsection{Weather sondes}

The data were acquired at the Dome $\mathrm{C}$ station $\left(75^{\circ} 06^{\prime} \mathrm{S}\right.$ $123^{\circ} 19^{\prime} \mathrm{E}$ ) using balloon-borne weather sondes (model RS80 and RS90) manufactured by Vaisala. The sonde measures wind speed and direction by GPS triangulation as well as temperature, pressure and humidity from its in-built sensors. The humidity data will not be presented in this paper due to inconsistencies between the two types of sondes and their inaccuracies at this temperature regime.

The data were taken between November and February over four seasons (2000-2004) with a good statistical coverage of all sun zenith angles. Figure 1 shows the daily and monthly distribution of the sample used in this paper, consisting of a total of 145 successful balloons launches. The inversion layer, which is usually very low at Dome C, has been further sampled using captive sondes. These measurements were motivated both by the irregularity of the temperature data within the first few tens of meters of the balloon launch and by the importance that this part of the atmosphere has to astronomical site testing. The boundary layer is typically a major contributor to the seeing. It is therefore crucial to obtain numerous and accurate measurements of the temperature and wind profile of the low troposphere. The captive sonde temperature data were obtained by attaching the sonde to a pulley and raising it up to the top of a $30 \mathrm{~m}$ tower. The sonde was then slowly pulled down while the measurements were taken.

As each balloon explodes at a different height, the analysis of the next sections precludes data above altitudes for which the statistical noise dominates because the number of sonde data is low. This altitude varies depending on the type of data and the time span of the particular analysis. Typically, the analysis range reaches to $16 \mathrm{~km}$ and $20 \mathrm{~km}$.

\subsection{AWS}

For more than 23 years Automatic Weather Stations (AWS) have recorded data in Antarctica. The AWS are operated by the Antarctic Meteorological Research Center in Madison Wisconsin. The first AWS that we discuss in this paper was deployed to Dome C (74.50S, 123.00E, $3280 \mathrm{~m})$ in 1980. In $1995 / 1996$ the AWS was moved some $70 \mathrm{~km}$ to a new location known as Dome CII (75.12S, 123.37E, $3250 \mathrm{~m})$, from where it has operated ever since.

Each AWS samples the data approximately once every ten minutes. These instantaneous values are compiled by the Antarctic Meteorological Research Center and made publicly available on their website (http:// uwamrc.ssec.wisc. edu). Over the past 20 years the reliability of AWS data has improved considerably. Error rates, which

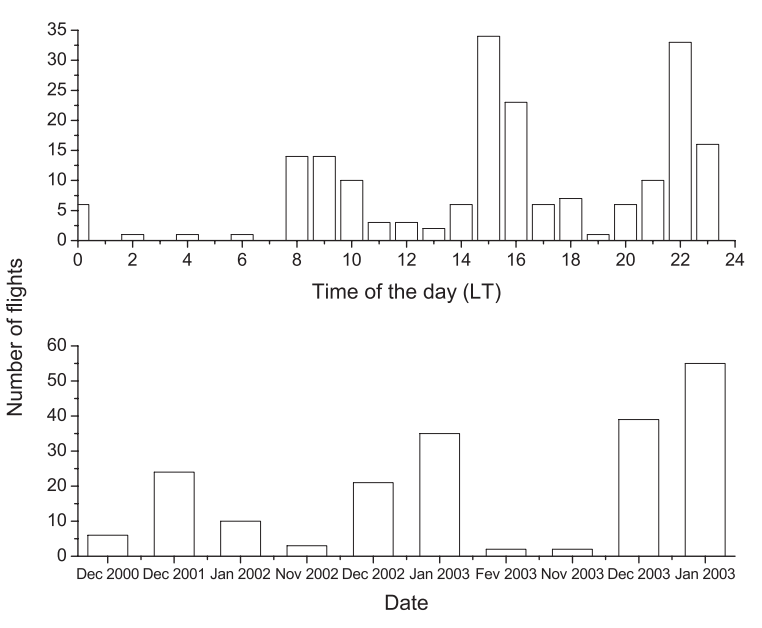

Fig. 1. Time distribution of the measurements as a function of hour of the day and month.

used to be as high as $71 \%$, are now typically around $20 \%$. In addition, some stations now measure humidity as well as the standard quantities of wind, wind direction, pressure and temperature. The temperature sensor is installed $3 \mathrm{~m}$ above the ground. The wind sensor is mounted around $1 / 3$ of a meter above that.

Since 2001, single "anomalous" wind speed readings are registered with peaks of up to $62.2 \mathrm{~m} / \mathrm{s}$. These apparent gusts are completely non-physical and arise from recording errors in the AWS. Prior to 2001 they were automatically removed before the data were publicly released. However, as a result of a changed software procedure by the Antarctic Meteorological Research Center, these data are no longer removed. We have therefore set up a "clipping" level of $18 \mathrm{~m} / \mathrm{s}$ and removed any single wind speed peak that is above this level. The highest "real" peak (having a steadily increasing wind speed before and a steadily decreasing wind speed after) occurred in July 1988 with a speed of $20.2 \mathrm{~m} / \mathrm{s}$.

\section{Results}

In the following sections, the height is defined with respect to the Dome $\mathrm{C}$ ice level rather than the altitude above sea level (unless specified).

\subsection{Wind speed and direction}

On the Antarctic continent, the wind profile is characterized by two phenomena. At the surface, katabatic winds descend from the high plateau and increase in speed as they reach the coast. Similar to inversion winds, their speed is closely related to the slope of the local terrain. At Dome C, where the slope is near zero the ground speed is very low. In Fig. 2 we present the mean wind speed for each month from the beginning of 1984 to the end of 2003.

There is a clear difference between the average wind speed recorded at Dome C and that at Dome C II, the latter being higher by $0.5 \mathrm{~m} / \mathrm{s}$. It is not known to what extent this is a real effect, and to what extent it simply reflects a difference in calibration between the two weather stations. For neither station is there any evidence of a trend that would suggest wind 


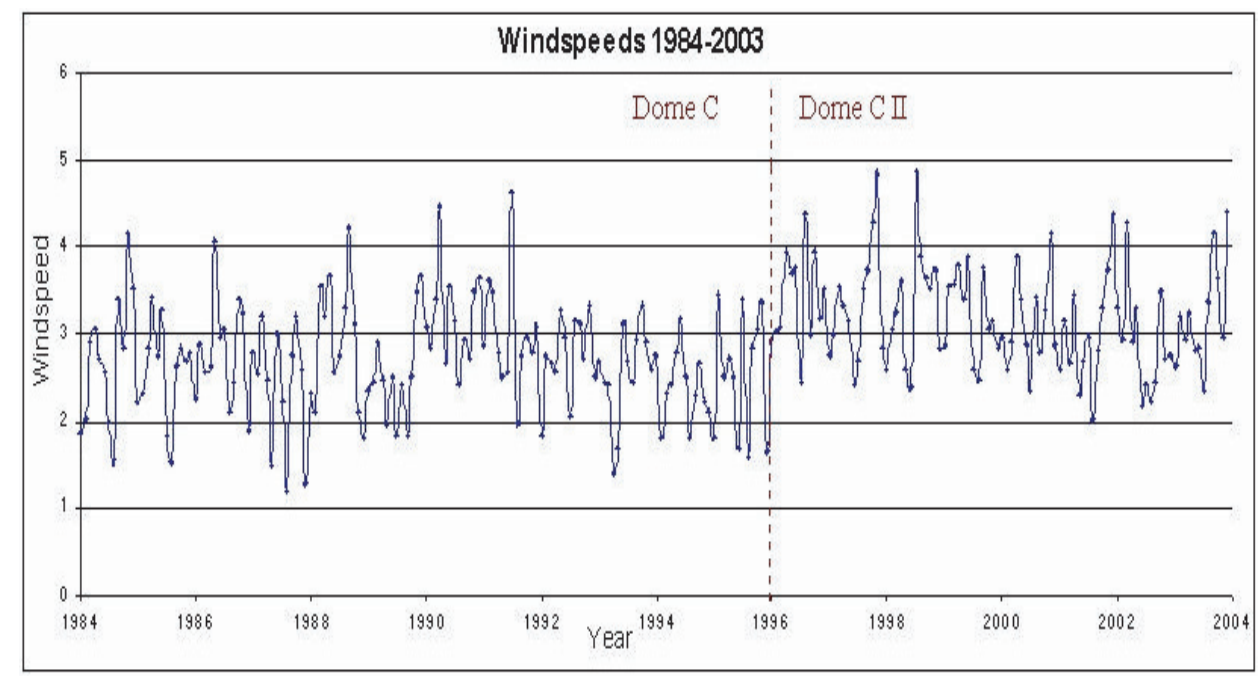

Fig. 2. Mean monthly wind speed at Dome C and Dome C II from the beginning of 1984 to the end of 2003.

Table 1. Mean wind speeds at Dome $\mathrm{C}$ and other astronomical sites for which long-term data exist.

\begin{tabular}{ccc}
\hline \hline Site & Wind speed $(\mathrm{m} / \mathrm{s})$ & Reference \\
\hline Dome C (1984-1995) & 2.7 & This paper \\
Dome C II (1996-2003) & 3.2 & This paper \\
Dome C plus Dome CII (1984-2003) & 2.9 & This paper \\
Maidanak & 2.1 & Ehgamberdiev et al. (2000) \\
Mauna Loa & 4.4 & Barnes (2004) \\
La Silla & 4.6 & Hainaut (2004) \\
South Pole & 5.5 & Mefford (2004) \\
La Palma & 6.6 & La Palma (2004) \\
Paranal & 6.6 & Hainaut (2004)
\end{tabular}

speed is increasing with time. Using the combined data taken from Dome C and Dome C II, we obtain a mean wind speed of $2.9 \mathrm{~m} / \mathrm{s}$. This is the lowest wind speed recorded at any major or planned site with the exception of Maidanak (see Table 1). Antarctic sites located on a local slope, such as Vostok or South Pole, experience greater ground level winds ( 5.1 and $5.8 \mathrm{~m} / \mathrm{s}$ respectively, Schwerdtfeger 1984) because of the inversion and katabatic winds. In Table 2, we present some representative data. We show data for the first and last years of the period analysed (1984 and 2003), plus data at 6-year intervals (1990, 1996,2002 ) in order to look for possible long-term trends.

In each case we present data for the whole year (typically 15000 data points for Dome C and 40000 data points for Dome CII), for the 6 months of winter (22 March-22 September with typically 8000 data points for Dome C and 20000 data points for Dome CII), and for those periods when the sun is 18 degrees or more below the horizon (astronomical darkness) with typically 3500 data points for Dome C and 9000 data points for Dome CII). In general, as we enter the winter months the mean and median wind speeds both decrease, and the percentage of time for which there is zero wind increases. In other words, those observing times that are of greatest interest to optical astronomers are also those with the most favourable winds.
Table 2. Median wind speeds in $\mathrm{m} / \mathrm{s}$ at Dome $\mathrm{C}$ over the course of several years (see text).

\begin{tabular}{cccc}
\hline \hline Year & All data & Winter & Astronomical darkness \\
\hline 1984 & 2.4 & 2.1 & 1.9 \\
1990 & 2.9 & 2.9 & 2.9 \\
1996 & 3.0 & 3.0 & 3.0 \\
2002 & 2.5 & 2.5 & 2.2 \\
2003 & 2.8 & 2.8 & 2.5 \\
\hline
\end{tabular}

The results presented here suggest that the winds at Dome C are actually somewhat higher than reported by Valenziano \& Dall Oglio (1999). Those authors used data that had already been averaged into 3-h bins, which they further averaged to produce monthly data blocks. Additionally, they averaged the wind speed data to create $1 \mathrm{~m} / \mathrm{s}$ "bins". Their data also covered a smaller time interval (8 years; from 1986 to 1994). These factors probably explain why we find the wind speed to be higher than the $2.1 \mathrm{~m} / \mathrm{s}$ (median) reported in Table 2 of Valenziano \& Dall Oglio. Other figures for "average" and "50-percentile" wind speeds reported in that work do not appear to be self-consistent. We do, however, note that our Fig. 2 is in reasonable agreement with their Fig. 5. 


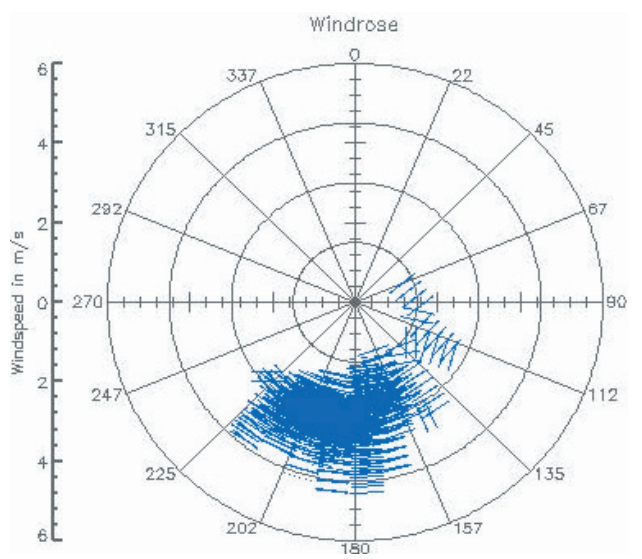

Fig. 3. Wind rose, showing the probability that the wind will be of a particular speed and in a particular direction, for Dome $\mathrm{C}$ and Dome C II combined, for the same period as in Fig. 2.

Being a point of origin, the prevailing wind is not as consistent as it is along the slope of the plateau (see Fig. 3). The prevailing inversion wind points North, however, towards the coastal base of Dumont D'Urville and along the direction of greatest slope. Wendler et al. also report the wind conditions using several AWS between the two stations showing increasing wind speeds toward the coast and very consistent directionalities.

The wind speed profile (see Fig. 4) is ruled by the second phenomenon that characterises the wind conditions in Antarctica, the circumpolar vortex. Arising from a large temperature gradient between the coast and the open ocean, this quasi-perfect geostrophic wind circles the Antarctic continent. Highly decoupled from the boundary layer, the vortex is not centered on the South Pole but closer to the center of mass of the continent. In sub-polar regions, the speed of the vortex decreases with increasing latitude. In the troposphere, the seasonal variation of the vortex is minimum while in the stratosphere the vortex increases in speed in winter. This is demonstrated in Fig. 7. While winter-time data do not yet exist, our first measurement from mid-November show speeds of up to $40 \mathrm{~m} / \mathrm{s}$ at $20 \mathrm{~km}$ altitude. After early December the stratospheric wind drops and rarely exceeds $10 \mathrm{~m} / \mathrm{s}$. This behavior has also been observed at a latitude of $60^{\circ} \mathrm{S}$ (see Fig. 4.8, p. 132, in Schwerdtfeger 1984). To observe such a large stratospheric wind speed at the latitude of Dome $\mathrm{C}$ is unexpected and is likely a rare event triggered by the breaking of the vortex in 2002-2003, as suggested by Chanin (2003).

Figure 5 shows the wind statistics at $200 \mathrm{mB}$, corresponding to an altitude of $7 \mathrm{~km}$ above Dome $\mathrm{C}$. The wind speed at this altitude is often taken as a reference altitude in site testing. Usually corresponding to the mean turbulence-weighted altitude of several Chilean sites, it has been found that the wind speed at this altitude is inversely related to the coherence time of the site (Vernin 1986). While it remains to be shown that the mean turbulence altitude at Dome $\mathrm{C}$ is at $200 \mathrm{mB}$, we will show in section four that at least part of the turbulence is generated at this altitude. With a mean of $7.6 \mathrm{~m} / \mathrm{s}$, the summer time tropopause wind at Dome $\mathrm{C}$ is much lower than at typical mid-latitude sites during the same season. At

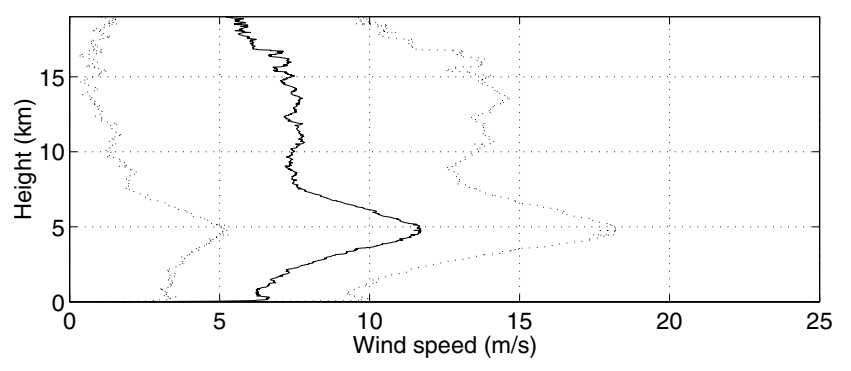

Fig. 4. Mean wind speed profile at Dome C. The two outer lines delimit the standard deviation.

Mauna Kea and Paranal, for example, the mean $200 \mathrm{mB}$ wind speeds in December are $24.7 \mathrm{~m} / \mathrm{s}$ and $22.2 \mathrm{~m} / \mathrm{s}$ respectively (Vernin 1986). While we do not have Dome C data in the middle of winter, it is known that at this latitude, the vortex does not normally vary greatly with seasons (see Fig. 4.8, p. 132, in Schwerdtfeger 1984).

A site testing campaign having been carried out at the South Pole station, a comparison of the wind conditions at the two sites is now possible. Table 3 gives the winds statistics of Dome $\mathrm{C}$ and South Pole. Note that the two data sets use AWS data for the ground values and cover the same period as the balloon data.

At ground level, the wind speed is lower at Dome C. This is explained by the difference of slope between the two sites. Located on a local maximum, Dome $\mathrm{C}$ is rarely affected by katabatic winds. At the South Pole the gentle slope drives the surface wind from higher points of the plateau. The very low average wind speed and the absence of strong gusts makes Dome C a very suitable location for the building of an observatory.

Up to an altitude of $14 \mathrm{~km}$ the wind direction is very stable, slowly moving from a south to a south-easterly direction (see Fig. 6). This direction follows the topological wind flow pattern described in Parish (1980). Above $14 \mathrm{~km}$, an abrupt change is observed, the wind moving rapidly clockwise. This effect is probably due to the sonde travelling north and eventually being caught by the stratospheric vortex that circles the continent in the same direction. As seen in Fig. 4, this sudden change of direction is not accompanied by a variation of the wind speed.

Compared to other well-known astronomical sites, the wind speed profile at Dome $\mathrm{C}$ is very encouraging. Figure 7 shows a typical summer (full line) and end of winter (in dash) profile at Dome $\mathrm{C}$ and at South Pole compared with typical profiles at Mauna Kea and Paranal. In the temperate sites, the jet stream is clearly observed at an altitude of $12 \mathrm{~km}$ where the most intense turbulence of the free atmosphere normally occurs. The Antarctic sites have very flat summer profiles with no presence of high altitude winds. Our first measurements taken in midNovember can be interpreted as a good indication of the winter conditions. Due to the presence of the stratospheric vortex, wind speeds show a broad peak at an altitude of $20 \mathrm{~km}$, substantially higher than at temperate sites. 
Table 3. Wind statistics at Dome C and South Pole over the period of time covered by the balloon launches.

\begin{tabular}{lllll}
\hline \hline & Dome C & & & \\
\hline & ground & trop. $-330 \mathrm{mB}$ & $200 \mathrm{mB}-11 \mathrm{~km}$ & $50 \mathrm{mB}$ \\
$N$ data & 8741 & 145 & 123 & 75 \\
Mean w speed & 3.6 & 12.2 & 7.4 & 6.6 \\
Med. w speed & 3.5 & 10.7 & 5.9 & 4 \\
W speed std dev & 2.5 & 7.2 & 5.3 & 6.1 \\
Max w speed & 10.5 & 41 & 25.7 & 34.4 \\
Mean direc. & 180.3 & 187.6 & 211.6 & 193 \\
Direc. std dev & 74.4 & 92.6 & 90.8 & 121.6 \\
\hline & South Pole & & & \\
\hline & ground & trop. $-330 \mathrm{mB}$ & $200 \mathrm{mB}-11 \mathrm{~km}$ & $50 \mathrm{mB}$ \\
$N$ data & 668 & 666 & 662 & 621 \\
Mean w speed & 4.7 & 10 & 7 & 9.2 \\
Med. w speed & 4.5 & 8.1 & 5.7 & 4.7 \\
W speed std dev & 2 & 7 & 4.9 & 9.6 \\
Max w speed & 13 & 47.8 & 35.4 & 56 \\
Mean direc. & 105.9 & 213.7 & 203.6 & 155.3 \\
Direc. std dev & 112.3 & 113.3 & 114.4 & 100.4 \\
\hline
\end{tabular}

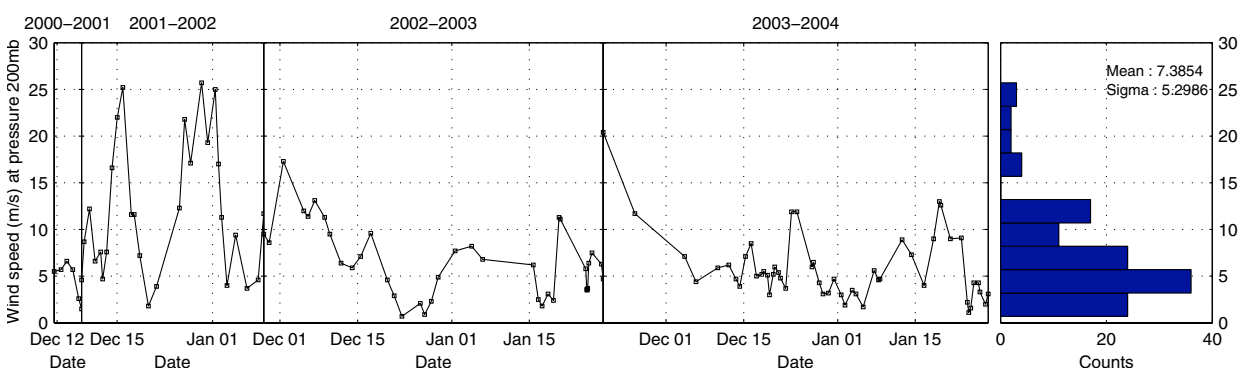

Fig. 5. $200 \mathrm{mB}$ wind speed distribution.

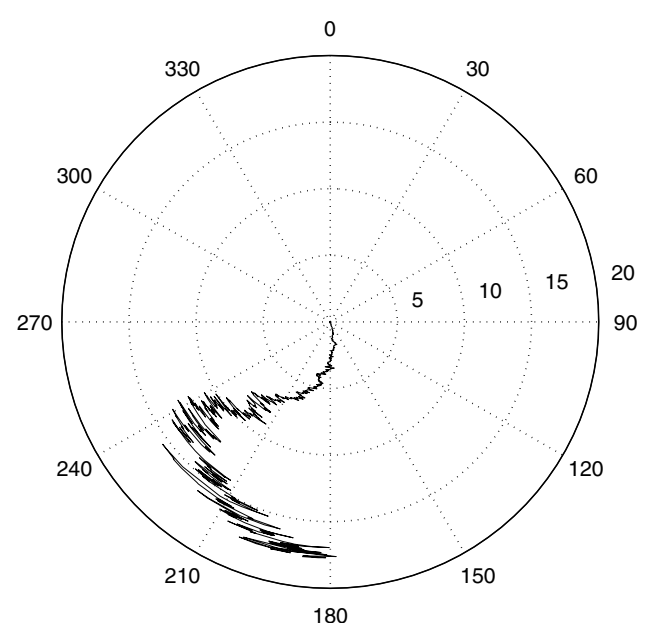

Fig. 6. Average wind direction profile as a function of altitude.

\subsection{Temperature}

The ground temperature at Dome $\mathrm{C}$ is typical of other widely reported inland sites such as Vostok and South Pole and was reported in (Schwerdtfeger 1984). The monthly averages are shown for both South Pole (from meteorological records given in the previous reference) and Dome $\mathrm{C}$ in Fig. 8 using the AWS data. At both sites the core-less winter represents about 6 months of the year of quasi-constant temperatures. The long period of extremely cold temperatures is remarkable for telescope sensitivity. It is interesting to note that while Dome $\mathrm{C}$ winter temperatures are lower because of the altitude difference, South Pole has the longest winter because of its latitude. A compromise must therefore be made between sensitivity and total observation time below a given sky brightness.

The average summer temperature profile for Dome $\mathrm{C}$ is presented in Fig. 9. The tropopause, defined by the minimum temperature gradient is found at a height of $5.5 \mathrm{~km}(330 \mathrm{mB})$ above the ice and followed by a very brief isothermal layer less them $1 \mathrm{~km}$ wide. This feature is known to be less evident in winter from South Pole observations when the stratosphere cools. An analysis by time period shown in Fig. 10a demonstrate the stability of the the troposphere temperature profile. Larger thermal fluctuations are observed in the stratosphere. More important to astronomical sites is the strength and depth of the inversion layer. This is particularly true at the South Pole, where a large majority of the seeing is generated within the boundary layer, as shown at the South Pole (Travouillon et al. 2003; 

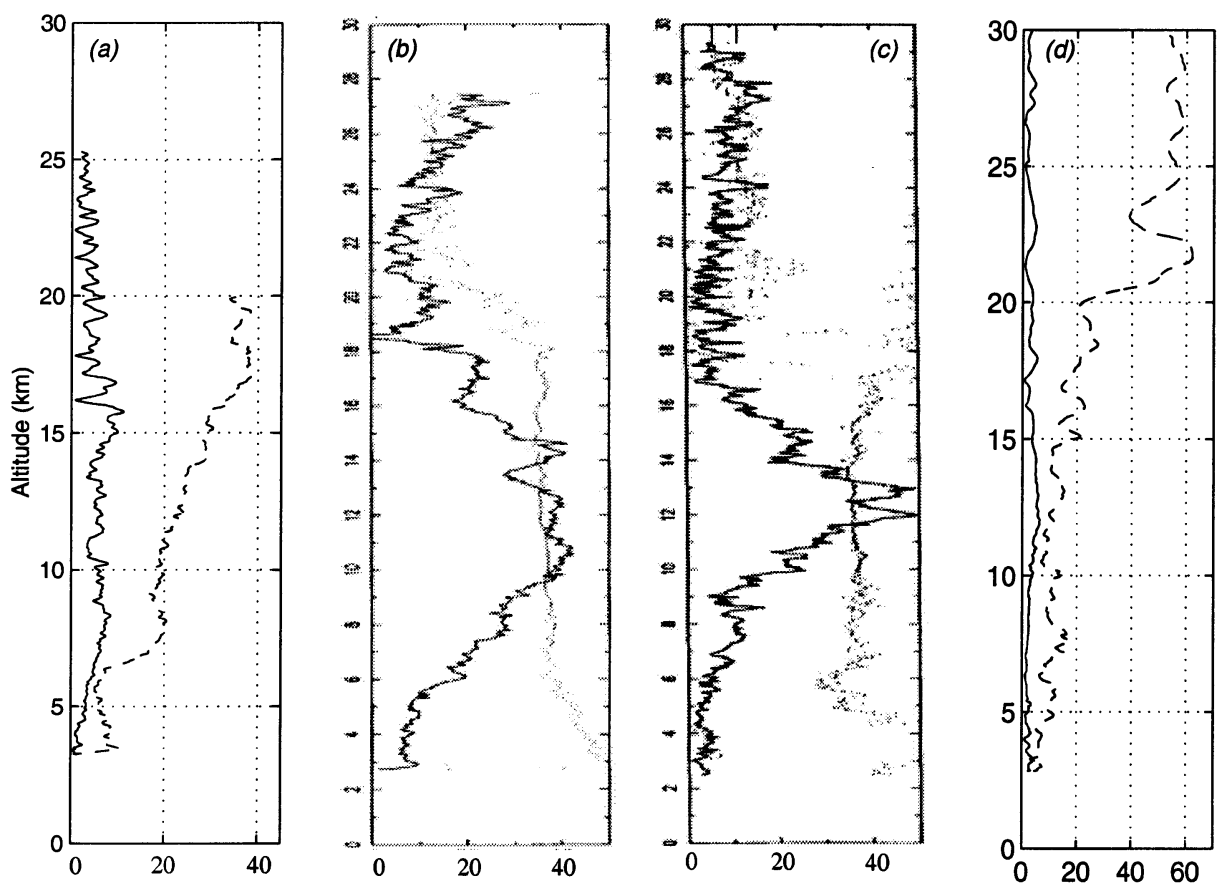

Fig. 7. typical wind speed profiles at a) Dome C, b) Paranal (Chile), c) Mauna Kea (Hawaii) d) and South Pole. In the case of the Dome C and South Pole, the profile in dashed line is representative of the end of winter while the full line is a typical summer profile. Note: the altitude is here expressed from sea level.

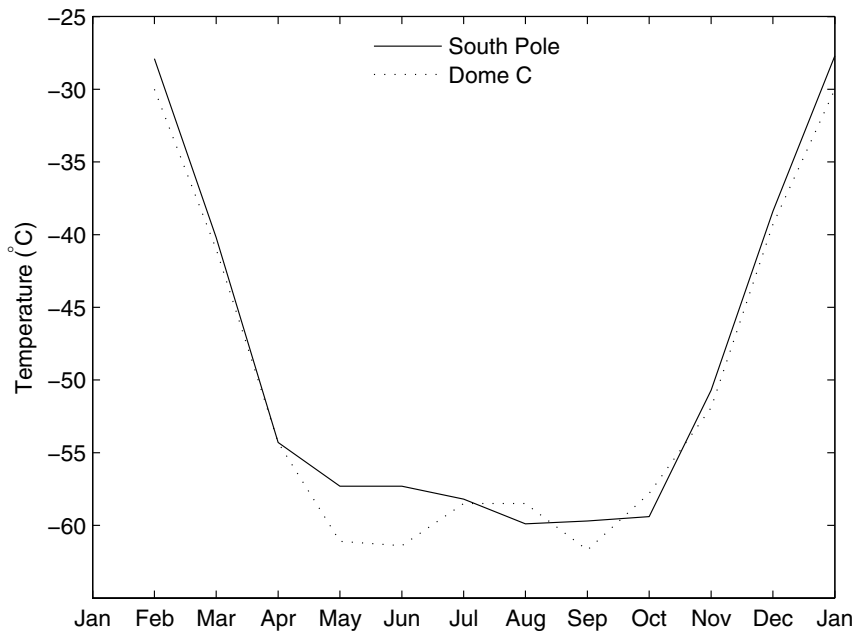

Fig. 8. Monthly average temperatures at South Pole (Schwerdtfeger 1984) and Dome C.

Marks et al. 1999). Figure 10b shows the inversion layer at Dome $\mathrm{C}$ as a function of time of day. In winter we expect the inversion to resemble that of the summer night time measurement with a more pronounced temperature gradient. The important point to note is the narrow depth of the inversion. Extending to only $50 \mathrm{~m}$ this is much lower than the boundary layer at the South Pole (220 m, Marks et al. 1999) or Vostok (300 to $500 \mathrm{~m}$, King \& Turner 1997). While the seasonal variation of the inversion depth is unknown, it is unlikely that it will increase greatly due to the geographical location of Dome C. King \& Turner (1997) have measured larger variations on coastal stations than inland ones.

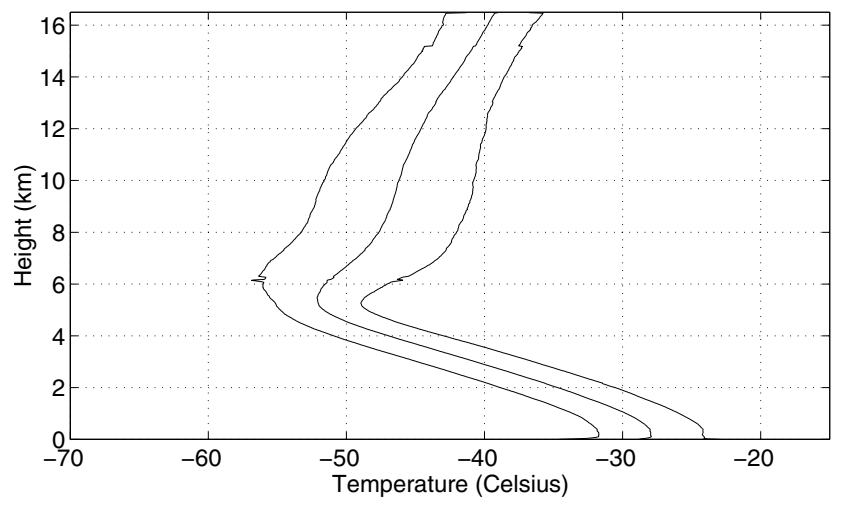

Fig. 9. Mean temperature profile. The two outer lines delimit the standard deviation.
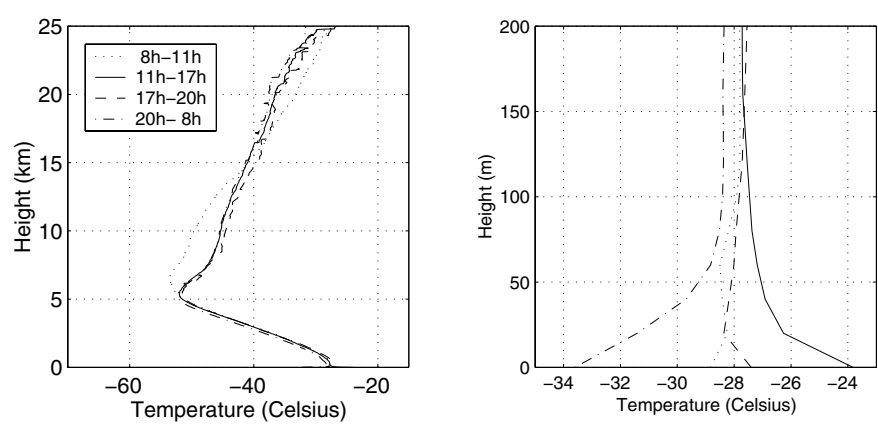

Fig. 10. Temperature profiles for different time periods. The second graph focuses on the inversion zone.

In order to further investigate the temperature profile of the boundary layer, we have launched balloons at two-hourly intervals during two days of 2003 and 2004. We have chosen 

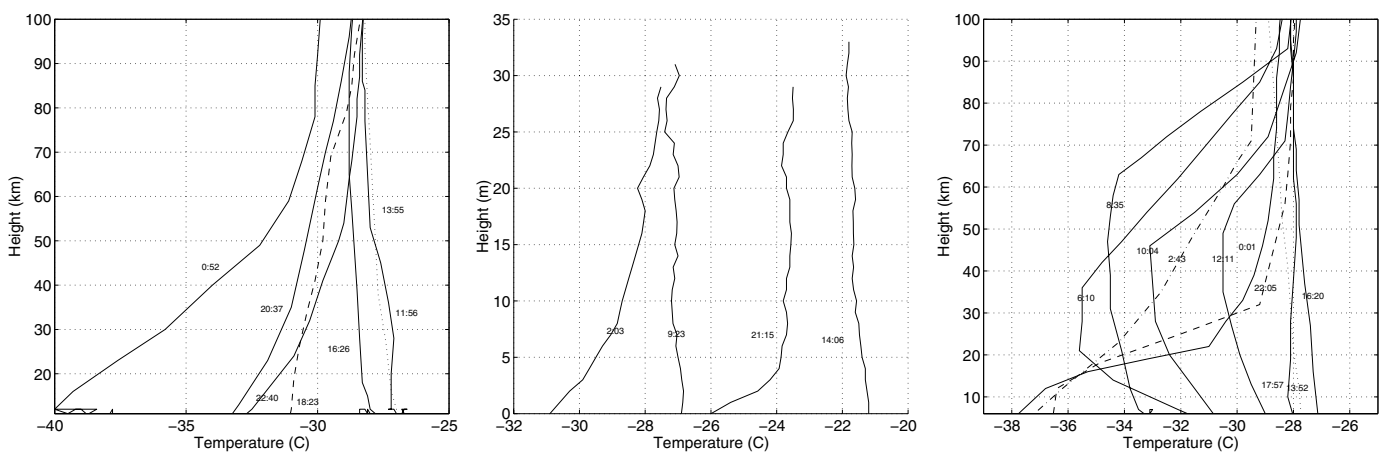

Fig. 11. Left: temperature profiles taken at regular intervals on the 25/01/03; center: temperature profiles representative of the indicated hour measured using a pulley; right: temperature profiles taken at regular intervals on the 24/01/04.

the two days to be almost exactly a year apart to make the data more comparable. Figure 11 shows the temperature profiles of these two days as well as representative data measured using sensors pulled down from the $30 \mathrm{~m}$ tower.

Balloon borne sondes can measure up to ten point in the first $30 \mathrm{~m}$ while the number of point obtained from the tower experiment is only limited by the speed at which we pull the sonde (we typically obtained 4 points per meter). The results show that the two methods are in good agreement. The inversion starts to become apparent at roughly 8:00 pm and remains trapped below $30 \mathrm{~m}$ until midnight where the largest gradient is observed in the first $20 \mathrm{~m}$. It then rises in altitude reaching a maximum of $90 \mathrm{~m}$, its gradient losing in intensity. Finally the inversion dies out as the sun warms the surface and equilibrates the surface layer at around $2 \mathrm{pm}$.

\section{Discussion and conclusion}

Wind speeds at Dome C are extraordinarily low, particularly in view of the fact that it is the highest point for several hundred kilometers. Both peak and average wind speeds are less than half that at most other sites, delivering a major advantage to both telescope designers and astronomers. For a substantial fraction of time the wind speed at Dome C is zero; in 1990, for example there was no wind at all for $6 \%$ of the darkest hours of the year. (An even larger fraction of zero-wind time occurs in 1984; however this appears to be an anomaly.)

It is, however, the combination of a low boundary layer and a remarkably stable free atmosphere that makes Dome C such a strong candidate for future large observatories. The mid-infrared sky brightness at Dome $\mathrm{C}$ one or two orders of magnitude lower than observed at typical mid-latitude sites (Walden et al. 2004) and the sub-millimeter opacity is significantly lower than at mid latitude sites and comparable to the South Pole (Calisse et al. 2004). A thorough study of the turbulence profile is necessary to determine the performance that can be obtained with and without adaptive optics.

Preliminary turbulence information can be inferred from the meteorological data. The computation of the Richardson number throughout the atmosphere is a good indicator of the generation of optical turbulence and of its location.
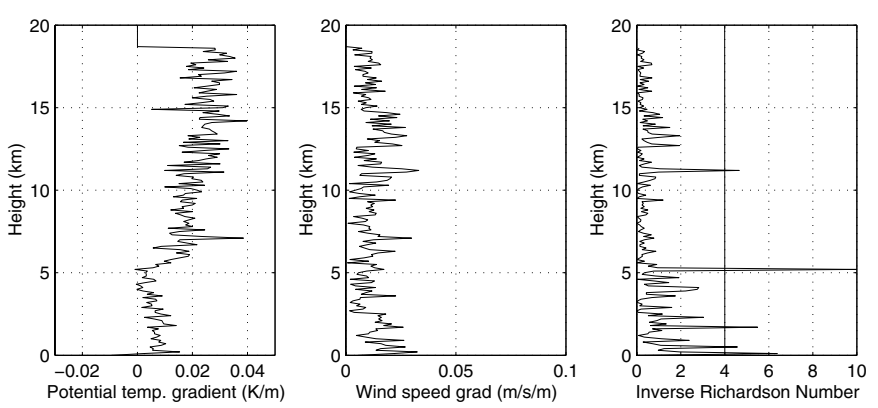

Fig. 12. Temperature gradient, wind speed gradient and Richardson number profile for a typical data set.
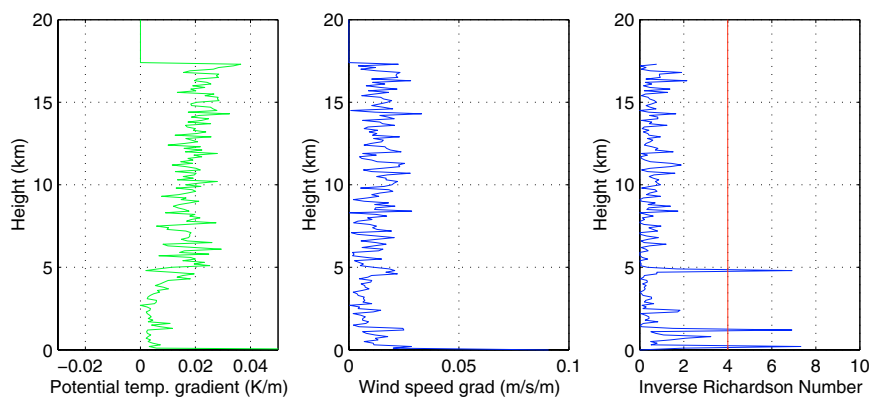

Fig. 13. Temperature gradient, wind speed gradient and Richardson number profile for a typical data set.

The following criterion is usually stated as a condition of formation of turbulence:

$R_{i}=\frac{g}{\theta} \frac{(\mathrm{d} \theta / \mathrm{d} z)}{\left.(\mathrm{d} U / \mathrm{d} z)^{2}\right)}<\frac{1}{4}$

where the Richardson number, $R_{i}$, is an indicator of layer stability if non-negative, $g$ is the gravitational constant and $\mathrm{d} \theta / \mathrm{d} z$ and $\mathrm{d} U / \mathrm{d} z$ are the potential temperature and wind speed gradients with respect to altitude. Potential temperature is defined as:

$\theta=T\left(\frac{1000}{P}\right)^{0.286}$

where $T$ is in Kelvin and $P$ in millibar. Wind speed gradient is

$\frac{\mathrm{d} U}{\mathrm{~d} z}=\sqrt{\left(\frac{\mathrm{d} U_{x}}{\mathrm{~d} z}\right)^{2}+\left(\frac{\mathrm{d} U_{y}}{\mathrm{~d} z}\right)^{2}}$ 
with $U_{x}$ and $U_{y}$ the two components of the wind speed vector. Gradients are computed as follows: altitudes are sampled with $100 \mathrm{~m}$ interval in which a linear interpolation of $\theta(z)$, $U_{x}(z)$ and $U_{y}(z)$ is performed. Calculated slopes are taken as the gradients. In Figs. 12 and 13 we have plotted two typical gradient profiles as well as the inverse of the Richardson number. The vertical line (at 4) indicates the limit beyond which turbulence can be generated. It was found that turbulence was almost systematically present in the boundary layer during periods of inversion due to the sharp temperature gradient. In the free atmosphere, however, conditions of turbulence generation are few and spatially thin. The only peak found commonly in the free atmosphere is usually found between 5 and $7 \mathrm{~km}$ corresponding to the top of the tropopause. These results also show that some turbulence is generated in the boundary layer. This is expected as the measurements are taken in daytime where the boundary layer is usually convective. In winter the boundary layer turbulence will be different and it will necessary to determine intensity and the spatial extent of the boundary layer turbulence.

The inescapable conclusion is that Dome $\mathrm{C}$ offers a remarkable opportunity for the construction of large and novel telescopes thanks to the excellent wind conditions and atmospheric stability (Lardiere et al. 2004; Angel et al. 2004). The average ground wind speed and the absence of violent "gusts" are very advantageous to the structural design of an observatory at Dome C. Recent nighttime site testing results (Lawrence et al. 2004) also confirm that the seeing is better than at temperate sites. The thermal stability and low wind speed in the free atmosphere present the ideal conditions for the use of adaptive optics. Hence, we have a combination of factors that must motivate further astronomical development at Dome C. It must not be forgotten, however, that even better conditions may still be found in Antarctica. The summit of the Antarctic plateau, Dome $\mathrm{A}$, is at an altitude exceeding $4000 \mathrm{~m}$ and we expect it will house the best observing conditions on the planet.

Acknowledgements. We would like to thank the Dome C staff for the logistic support as well as Steve Warren for the very helpful discussions. We would like to equally acknowledge the South Pole meteorological team for the South Pole data. Wind data were provided by the Automatic Weather Station run by the Antarctic Meteorological
Research Center in Madison, Wisconsin, USA. We thank Linda Keller and her team for their excellent support, and their assistance to us in understanding the data. Financial and logistic support was provided by ARC, IPEV, PNRA and NSF.

\section{References}

Angel, R., Lawrence, J. S., \& Storey, J. W. V. 2004, Proc. of the 2nd Bäckaskog Workshop on Extremely Large Telescopes, Bäckaskog Castle, Sweden, 9-11 September 2003, 406, 19

Aristidi, E., Agabi, A., Vernin, J., et al. 2003, A\&A, 406, 19

Barnes, J. E. 2004, private communication

Calisse, P. G., Ashley, M. C. B., Burton, M. G., et al. 2004, PASA, 21, 256

Chanin, M.-L. 2003, An exceptional situation in the Antarctic stratosphere in 2002, in The scientific outlook for Astronomy and Astrophysics Research at the CONCORDIA station: Recommendations for future developments

Ehgamberdiev, S. A., Baijumanov, A. K., Ilyasov, S. P., et al. 2000, A\&AS, 145, 293

Hainaut, O. 2004, http://www . eso.org/astclim/lasilla

King, J. C., \& Turner, J. 1997, Antarctic Meteorology and Climatology, Atmospheric and Space Science Series (Cambridge)

Lardière, O., Salinari, P., Jolissaint, L., et al. 2003, Workshop on Extremely Large Telescopes, Bäckaskog Castle, Sweden, 9-11 September, Proc SPIE, in press

Lawrence, J. S., Ashley, M. C. B., Tokovinin, A., \& Travouillon, T. 2004, Exceptional astronomical seeing conditions above Dome C in Antarctica, Nature, in press

La Palma 2004, http://www.not.iac.es/cgi-bin/weather-archive.pl

Marks, R. D., Vernin, J., Azouit, M., Manigault, J. F., \& Clevelin, C. 1999, A\&AS, 134, 161

Mefford, T. 2004, NOAA CMDL, http: //www. cmdl . noaa.gov

Schwerdtfeger, W. 1984, Weather and Climate of the Antarctic, Developments in atmospheric science, 15 (Elsevier)

Travouillon, T., Ashley, M. C. B., Burton, M. G., Storey, W. V., \& Loewenstein, R. F. 2003, A\&A, 400, 1163

Valenziano, L., \& Dall'Oglio, G. 1999, PASA, 16, 167

Vernin, J. 1986, Proc. SPIE, 628, 142

Walden, V., Halter, B., \& Storey, J. W. V. 2004, PASP, submitted

Wendler, G., Andre, J.-C., Pettre, P., Gosink, J., \& Parish, T. 1992, Katabatic winds in Adelie coast, in Antarctic Meteorology and Climatology: Studies based on Automated Weather Stations, Antarctic Research Series, 61, 23 